\title{
POPULATION DYNAMICS OF COLLETOTRICHUM GLOEOSPORIOIDES ON DIFFERENT PARTS OF AN AVOCADO ( PERSEA AMERICANA) TREE
}

\author{
ANJANI M. KARUNARATNE ${ }^{1 *}$, R.O.THATTIL ${ }^{2}$ and N.K.B. ADIKARAM ${ }^{1}$ \\ ${ }^{1}$ Dcpartment of Botany, Faculty of Science, University of Peradeniya, Peradeniya. \\ "Department of Crop Science, Faculty of Agriculture, University of Peradeniya.
}

(Received: 11 August 1998 ; accepted: 03 September 1999 )

\begin{abstract}
Colletotrichum gloeosporioides in different parts of a single avocado tree, and its debris under the tree, was enumerated at monthly intervals, during a period of two years. A positive correlation between quiescent infections-QI on flowers and rainfall ( $R F)$, and a negative correlation of $Q I$ on twigs and $R F$ were observed. The positive correlation between $\mathrm{RF}$ and $\mathrm{QI}$ on leaves was significant only when RF was pre-lagged by 3 months, and the level of significance increased progressively when RF was pre-lagged upto 6 months. Disease symptoms were never observed on the vegetative parts of the tree and flowers, but a high number of QI was observed on twigs and leaves. This is probably how (. gloeosporioides survives between fruiting seasons. The different relationships between $\mathrm{QI}$ on each part of the tree and RF, demonstrate how this pathogen may have adopted itself to survive on the tree. Therefore the results suggest that once the $C$. gloeosporioides propagules find their way to a tree, the pathogen remains there throughout its life time causing anthracnose on fruits. There was a significant drop in the density of QI of C. gloeosporioides on larger, mature fruits compared to that of smaller, immature fruits. Throughout this study, the free-living viable fungal propagule counts of debris under the tree varied from $10^{1}$ to $10^{3} \mathrm{CFU} / \mathrm{g}$ while those of washings of leaves, twigs and flowers varied fiom 0 to $10^{2} \mathrm{CFU} / \mathrm{g}$.
\end{abstract}

Key words: Anthracnose, avocado, Colletotrichum gloeosporioidles.

\section{INTRODUCTION}

The mechanism of infection of avocado (Persea americana Miller) by Colletotrichum gloeosporioides (Penz.), the causal agent of anthracnose disease ${ }^{1,2}$, and the biochemical basis of resistance of unripe avocado fruit to the pathogen ${ }^{3,4}$ have been well documented. This disease is one of the major reasons for the reduced shelf-life of avocado fruits in many parts of the world ${ }^{2,5}$ and in Sri Lanka ${ }^{6,7}$.

As far back as 1971, it was demonstrated that decay spots could appear on softening fruits even if they have been inoculated with C. gloeosporioides as early as 3 months prior to harvest ${ }^{1}$. Seven years later it was observed that infections with C. gloeosporioides could occur on fruits from fruit set to harvest ${ }^{\mathrm{s}}$. More recently it was shown, that the fungus was able to remain quiescent on the avocado fruit for periods of at least 6 months".

\footnotetext{
* Corresponding author
} 
In the literature there are no records of the presence of quiescent infections (QI) of C. gloeosporioides on the vegetative parts of the avocado tree. However in mango, it is reported that $C$. gloeosporioides survived actively in diseased leaves, defoliated branch terminals and old inflorescences ${ }^{10}$.

The present study was carried out on a single avocado tree. This was only an initial attempt to identify the intrinsic and extrinsic parameters to focus on, in studying the means of survival of $C$. gloeosporioides in between fruiting seasons, and in understanding its infection pattern on the developing avocado fruit.

\section{METHODS \& MATERIALS}

The avocado tree used for sampling : A Persea americana Miller tree of Carribean origin, which is about 15 years old, approximately $20 \mathrm{ft}$ tall and healthy, growing in a home garden in Kandy with no special attention given, was studied. The other trees within a $10 \mathrm{~m}$ radius were a mango tree and a jak tree. The avocado tree was well exposed to the sun and the wind. Several other avocado trees were present within a $100 \mathrm{~m}$ radius, and the tree sampled was the healthiest and robust of them all.

Enumerating inoculum of $C$. gloeosporioides on avocado leaves, twigs, flowers and debris under the tree: Samples of leaves, pieces of young green twigs, flowers (during seasons) from the tree, and debris under the tree were collected at monthly intervals, and analysed for $C$. gloeosporioides counts immediately. This was done for a period of two years from 21 July 1991 and ending on 26 July 1993, where a total of 25 sets of counts for leaves and twigs, and 15 counts for flowers were taken. Samples of branches about $1 \mathrm{~m}$ in length from at least five randomly selected locations around the lower region of the trunk were cut by standing under the tree using a knife fixed to a long pole. Samples of debris under the tree were collected into a clean plastic bag at the same time the branches were collected. This consisted of a mixture of dry leaves, twigs and flowers. The samples were taken to the laboratory and the branches were kept fresh by immersing the cut end in water.

Estimation of QI: Approximately $2 \times 2 \mathrm{~mm}^{2}$ pieces of green tender peelings of twigs to a depth of approximately $1 \mathrm{~mm}$, leaves or fruit peel of about $1 \mathrm{~mm}$ thick were cut and surface sterilized by immediately dipping in $1 \% \mathrm{NaOCl}$ solution for $2 \mathrm{~min}^{11}$. Individual flowers were split in two before subjecting to surface sterilization. All surface sterilized tissues were plated on Cook's No. 2 agar medium $^{12}$ (containing $200 \mathrm{ppm}$ tetracycline). All culture plates made in these experiments were incubated at room temperature $\left(27 \pm 3^{\circ} \mathrm{C}\right)$ for 48 to $72 \mathrm{~h}$ and the emergence of $C$. gloeosporioides colonies from the plated tissue was taken as indicative of quiescent infections. Every month, one hundred tissue pieces from each part of the avocado tree (twigs, leaves, flowers when available) were taken 
randomly (a total of 300 pieces a month, when flowers were available, or 200 pieces when the tree was not in flower), so that the pieces represented all the cut branches. The plating of all the samples was completed within 3 days of collection.

Estimation of free living viable fungal propagule (FLVFP) counts of C. gloeosporioides: Standard dilution plate technique was used. Randomly obtained pieces (approx. $2 \times 2 \mathrm{~cm}^{2}$ ) of leaves, and twigs ( $\leq 5 \mathrm{~cm}$ length of approx. 3mm diameter), which made upto $10 \mathrm{~g}$ fresh weight separately, representing all the branches cut as described above, and debris (10g of a mixture of decaying twigs and leaves, of sizes described above) under the tree were separately placed in $90 \mathrm{ml}$ of sterile distilled water in a $250 \mathrm{ml}$ capacity Erlenmeyer flask. The flasks were shaken vigorously for approximately 2 min after placing a previously sterilized rubber lid, and $1 \mathrm{ml}$ from each was transferred to test tubes containing $9 \mathrm{ml}$ sterile distilled water to prepare a series of 10 fold dilutions. One millilitre portions from the dilutions and the original suspension were pipetted out into separate petri dishes to which melted Cook's No. 2 agar medium containing 200 ppm tetracycline, was poured.

Plating was completed on the day the samples were obtained. The number of typical C. gloeosporioides colonies that grew on the agar plates (of no dilution to $10^{-2}$ ) after incubating at room temperature for $48-72 \mathrm{~h}$, were counted and the values were expressed as the number of Colony Forming Units (CFU) per gram fresh weight of each part of the avocado tree plated.

At least one representative $C$. glocosporioides isolate from each part of the tree (twigs, leaves and flowers) and debris in both QI estimation study, and in the FLVFP estimation study, were stored on agar slants at $5^{\prime \prime} \mathrm{C}$ until pathogenicity was established as described below.

Estimation of $Q I$ of $C$. gloeosporioides on fruits at different maturity stages: Fruits at 6 different maturity stages (distinguished by their size) from very young upto those in harvesting maturity were sampled to determine the quiescent $C$. gloeosporinides infections on them. Soon after plucking from the tree, the average volumes were determined by immersing the entire fruit in water and noting the amount of water displaced by each fruit. The surface area of each fruit was estimated as the two-thirds power of the fruit volume ${ }^{11}$. Each surface area value thus obtained was rounded off to the nearest integer. Pieces $(2 \times 2 \mathrm{~mm})$ of peel tissues were obtained for surface sterilization followed by plating in such a manner that five pieces per unit surface area of each fruit were considered as the number of pieces to sample from each fruit. Therefore the mean number of pieces obtained from each fruit was: 5 pieces from stage 1, 9 pieces from stage 2,12 pieces from stage 3,56 pieces from stage 4, 95 pieces from stage 5,125 pieces from stage 6 . At the first three stages of maturity, 5 fruits each were sampled, whereas at the last three stages of maturity 2 fruits each were sampled. The first three stages represent fruits plucked within six weeks of anthesis and the last three represent fruits at stages 6 weeks prior to harvest. 
Representative peel samples were obtained covering all sides of a fruit. These were surface sterilized and plated as described earlier. The plates were incubated at room temperature for $72 \mathrm{~h}$ and the number of $C$. gloeosporioides colonies developed were counted, and this value was divided by 5 to obtain the number of quiescent infections per unit surface area (i.e. density) of the fruit. In this way an average density value was obtained for the total number of replicate fruits at a particular maturity stage. The $C$. gloeosporioides isolates obtained were stored on agar slants at $5^{\circ} \mathrm{C}$ and their pathogenicity on fruits was established as described below.

Determining pathogenicity of isolated C. gloeosporioides cultures : C. gloeosporioides isolates obtained were subcultured on freshly prepared Cook's No.2 agar plates and allowed to incubate for $72 \mathrm{~h}$ at room temperature until the cultures were fully grown. The surface of healthy, mature but unripe avocado fruits were wiped with $70 \%$ ethyl alcohol. Suspensions of conidia (approx. $10^{5}$ per ml) were prepared with each isolate in $2 \%$ sucrose solutions. Drops $(20 \mu \mathrm{l})$ of these suspensions were placed on 5 spots on fruits in one row along the longitudinal axis. A second parallel row of drops was placed on 5 spots which were wounded by piercing the skin to a depth of $2 \mathrm{~mm}$ using a sterile needle. The inoculated fruits were kept inside moist chambers for $24 \mathrm{~h}$ and after that they were allowed to ripen in trays in the laboratory at room temperature unti] typical anthracnose lesions developed. The pathogen was reisolated into agar plates and the cultural characteristics were compared with the original isolates.

Rainfall (RF) was measured daily with the aid of a rain gauge (Yagami International Trading, Japan), during the two year period of analysis. The monthly RF was determined by totalling the daily values from the day after the previous month's sample was taken, to the day the present sample was taken. The RF values before the study started (i.e. in July 91) were determined by adding up the daily values for 30 day intervals (a total of 6 ) prior to the first sampling.

Statistical analysis : A five month moving average was used for the time series data of monthly RF and percentages of conidial counts of each part of the tree. The relationship between the independent variable (monthly $R F$ ) and each of the dependent variables (i.e. QI of percentage leaf, twig and flower) were

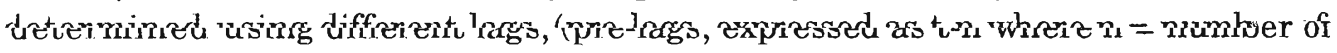
months) for RF. Regression analysis was performed for each of the dependant variables with $\mathrm{RF}$ at different lags and the most influential lags were determined. 


\section{RESULTS}

Flowering was observed from January to June of 1992 and from December 1992 to July 1993 with a freak flowering period in between in October 1992 (Figure 1). The estimated QIs fluctuated during this period of study and ranged from 3 to $93 \%$ on leaves, 13 to $86 \%$ on twigs, 1 to $95 \%$ on flowers. The total FLVFP counts on the tree were low but consistent throughout the two year period except in July and September 1991, when the counts on the tree as a whole, were zero (Table 1). Of the FLVFP counts taken, the highest were seen in washings of debris under the tree which varied from 0 to $10^{3} \mathrm{CFU} / \mathrm{g}$. The FLVFP counts of washings of leaves, twigs and flowers varied from 0 to $10^{2} \mathrm{CFU} / \mathrm{g}$. Unlike QIs, there were 6 occasions on leaves, 5 occasions on twigs and 6 occasions on flowers where there were no FLVFP of $C$. gloeosporioides detected. There was no instance where FLVFP counts on the tree and debris were zero in the same month (Table 1). The C. gloeosporioides count in debris was zero only on one occasion, which corresponded to a sudden increase of $\mathrm{RF}$ in October 1991.

The graphical plots of monthly RF and QI on leaves, twigs, and flowers are given in Figure 1. No significant relationships were observed between the monthly $\mathrm{RF}$ values and each of the dependent variables. Therefore, 5 month moving averages were taken to study the trends. When moving averages of each of the dependent variables were compared with that of $R F$ values (using different lags for leaf infections) the relationships listed in Table 2 were observed. There was a significant positive correlation between RF (with no lag) and QI of flowers. The regression coefficient between $\mathrm{RF}$ (pre-lagged 3 months, up to 6 months) and QI on leaves was also positive. However, QI of twigs correlated negatively with RF. These statistically significant relationships between $R F$ and each dependent variable are graphically represented in Figure 2 . The two flowering seasons including the freak flowering observed in October 1992, are depicted continuously along with the corresponding $R F$ values, disregarding the periods where flowering was absent, as the 5 month moving averages were determined in this manner. 
Table 1: Monthly rainfall (RF) and free-living viable fungal propagule(FLVFP) counts [in(CFU/g)x100] of the avocado tree.

\begin{tabular}{|c|c|c|c|c|c|c|}
\hline Time & $\mathrm{RF}(\mathrm{mm})$ & Leaf & Twig & Flower & Debris & Total on tree \\
\hline \multicolumn{7}{|l|}{1991} \\
\hline July & 4.6 & 0 & 0 & NFS & 22 & 0 \\
\hline Aug & 68.1 & 0.5 & 3 & NF & 3 & 3.5 \\
\hline Sept & 37.8 & 0 & 0 & $\mathrm{NF}$ & 11 & 0 \\
\hline Oct & 177.9 & 0 & 8 & $\mathrm{NF}$ & 0 & 8 \\
\hline Nov & 181.3 & 1.2 & 2.3 & NF & 3.3 & 3.5 \\
\hline Dec & 0 & 8 & 7 & NF & 27 & 15 \\
\hline \multicolumn{7}{|l|}{1992} \\
\hline Jan & 23.8 & 8 & 0 & 0 & 2.6 & 8 \\
\hline Feb & 0 & 0.7 & 4 & 0.5 & 1.4 & 5.2 \\
\hline Mar & 2 & 0.1 & 0 & 0 & 42.5 & 0.1 \\
\hline Apr & 191.6 & 0 & 1.7 & 0.2 & 7.4 & 1.9 \\
\hline May & 187.2 & 0.5 & 0.5 & 0.8 & 1.5 & 1.8 \\
\hline June & 226.7 & 0.4 & 0.4 & 0.5 & 14 & 1.3 \\
\hline July & 291 & 0.5 & 0.4 & $\mathrm{NF}$ & 16 & 0.9 \\
\hline Aug & 186.6 & 0.6 & 0.6 & $\mathrm{NF}$ & 1.2 & 1.2 \\
\hline Sept & 214.7 & 1 & 0.7 & NF & 2.5 & 1.7 \\
\hline Oct & 274.2 & 0 & 0.6 & 0.4 & 5 & 1.0 \\
\hline Nov & 327.7 & 1.6 & 0.9 & NF & 8 & 2.5 \\
\hline $\mathrm{Dec}$ & 81.1 & 5 & 0.4 & 0.5 & 13 & 5.9 \\
\hline \multicolumn{7}{|l|}{1993} \\
\hline Jan & 4.5 & 0.3 & 0.8 & 0.2 & 4.2 & 1.3 \\
\hline Feb & 10.7 & 6.5 & 0.5 & 0 & 30.5 & 7.0 \\
\hline Mar & 69.3 & 1.1 & 0.6 & 0 & 12.5 & 1.7 \\
\hline Apr & 208.2 & 0 & 1 & 0 & 5 & 1.0 \\
\hline May & 141.8 & 0.1 & 0.3 & 9 & 2.5 & 9.4 \\
\hline June & 813 & 1.2 & 0 & 0 & 0.6 & 1.2 \\
\hline July & 226.8 & 1 & 1.3 & 4.4 & 1.9 & 6.7 \\
\hline
\end{tabular}

$\S N F=$ No flowers 


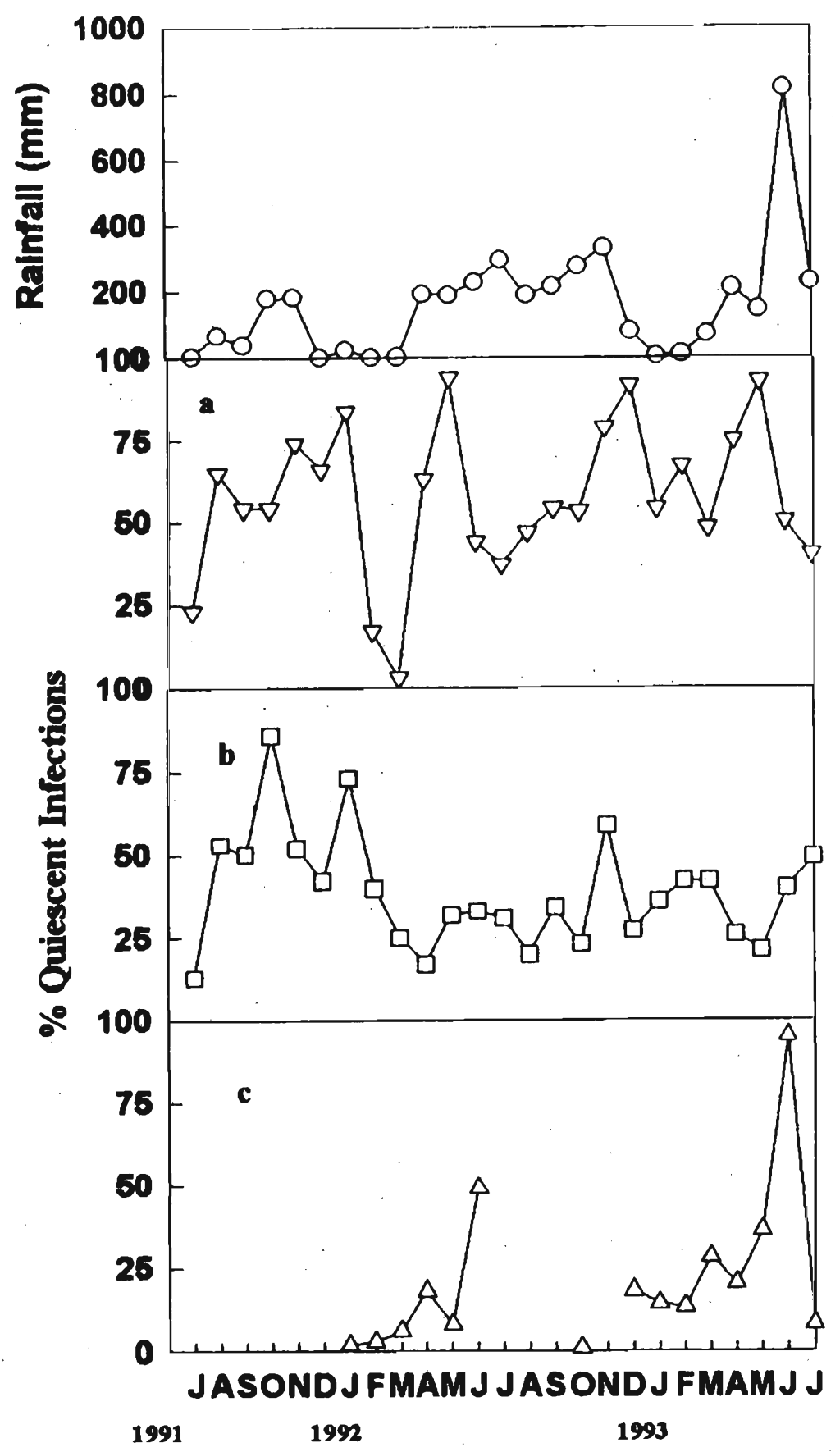

Figure 1: Monthly rainfall and counts of quiescent infections. The values for (a) leaves (b) twigs and (c) flowers (when in season) of avocado tree are expressed as a percentage during the period between July 1991 to July 1993. 
The results of the regression analysis performed between the dependent variables and RF are diagramatically depicted in Figure 3. The positive linear correlations between RF and QI of flowers, RF (pre-lagged t-3) and QI of leaves and the negative linear correlation between RF and QI of twigs are clearly depicted in these figures.

Table 2: Regression analyses of relationships between rainfall and percentage quiescent infections on flowers, leaves and twigs.

\begin{tabular}{lllll}
\hline $\begin{array}{l}\text { Variables } \\
\text { (QI) }\end{array}$ & $\begin{array}{l}\text { Significance } \\
\text { (P value for } \\
\text { correlation) }\end{array}$ & $\mathrm{R}$ & $\begin{array}{l}\text { Standard } \\
\text { deviation }\end{array}$ & Equation \\
\hline
\end{tabular}

Flower $(n=15)$

vs RF

0.003

0.78

0.025

$y=5.31+0.104 x$

Leaf $(n=25)$

vs $R F(t-3)$

0.024

0.45

0.023

$y=51+0.058 x$

vs $R F(t-4)$

0.025

0.44

0.024

$\mathrm{y}=50.9+0.058 \mathrm{x}$

vs RF (t-5)

0.002

0.61

0.022

$\mathrm{y}=48.6+0.076 \mathrm{x}$

vs RF (t-6)

0.000

0.73

0.018

$y=46.8+0.09 x$

Twig ( $n=25$ )

vs $R F(t)$

0.003

0.78

0.025

$y=49.7-0.076 x$

$\mathrm{t}=$ time. Pre-lags are expressed as $\mathrm{t}-\mathrm{n}$, where $\mathrm{n}=$ number of months.

$\mathrm{QI}=$ quiescent infections. $\mathrm{RF}=$ rainfall

QI counts of $C$. gloeosporioides on peels of fruits at different stages of maturity showed that the average conidial infections per unit area reduced sharply when the fruits enlarged with age (Figure 4). A Duncan multiple range test $(\mathrm{p}<0.05)$ revealed that the readings taken from the 3 immature stages of fruits were not significantly different from each other, and the readings of the fruits close to harvesting were not significantly different from each other. However these two sets of three readings each, were significantly different from each other.

Irrespective of the part of the tree from which the isolates was obtained, all C. gloeosporioides isolates tested caused typical anthracnose lesions on wound inoculated fruits. None of the inoculations made on unwounded skin of fruits developed into lesions. 


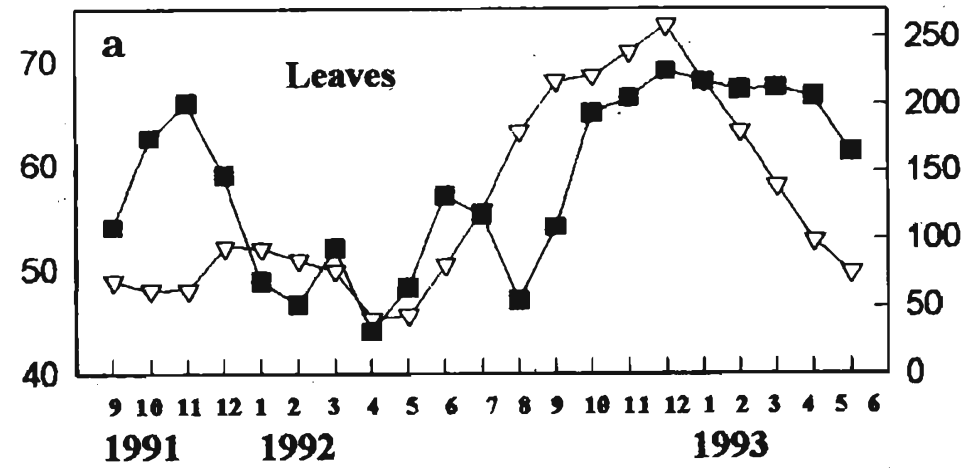

星
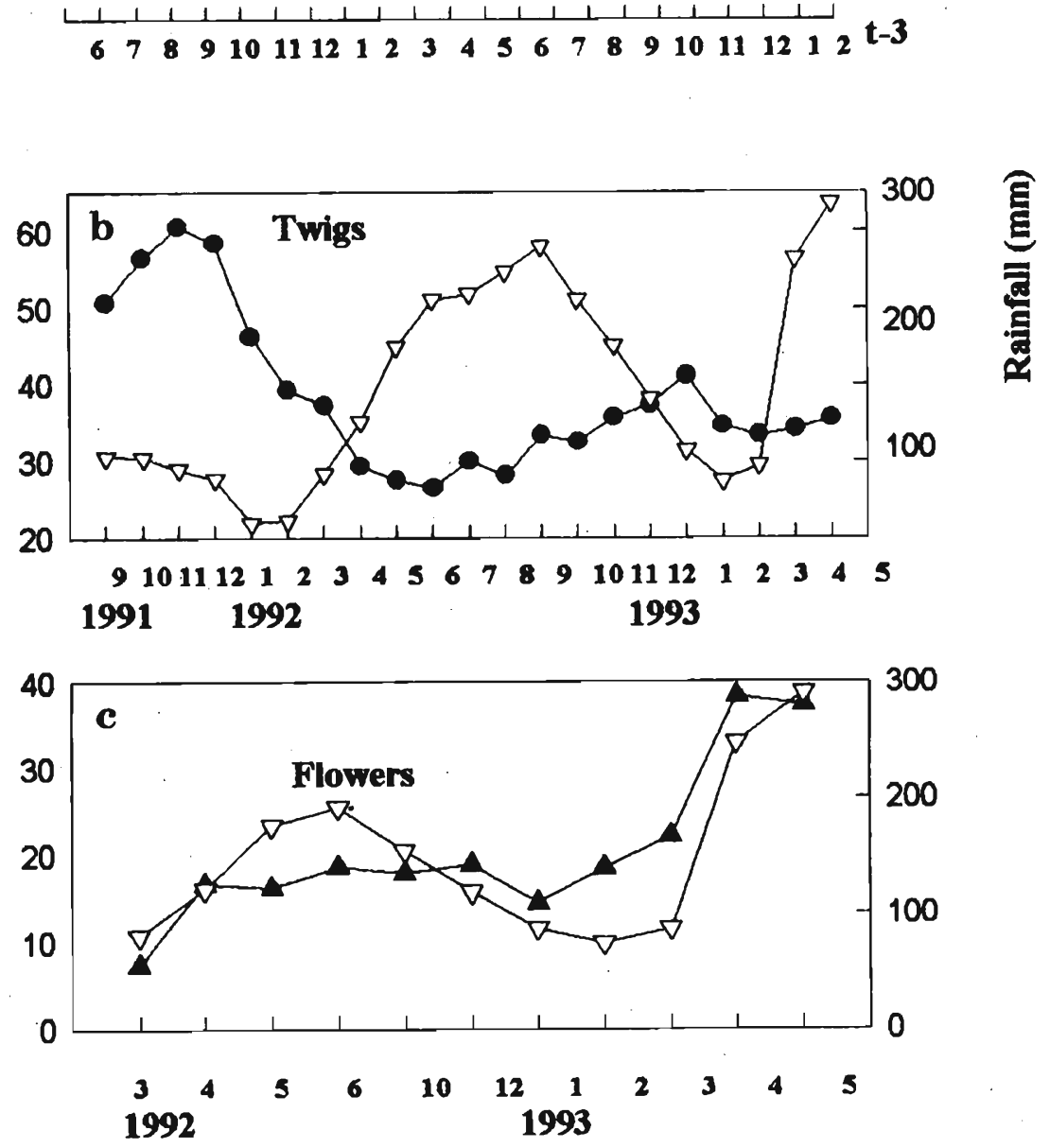

Figure 2: Graphical representations of relationships between rainfall and quiescent infections.

a) RF prelagged by 3 months (t-3) and QI of leaves, b) RF and QI of twigs, c) RF during flowering seasons and QI of flowers. Each 5 month moving average value is plotted centering to the 3rd month 

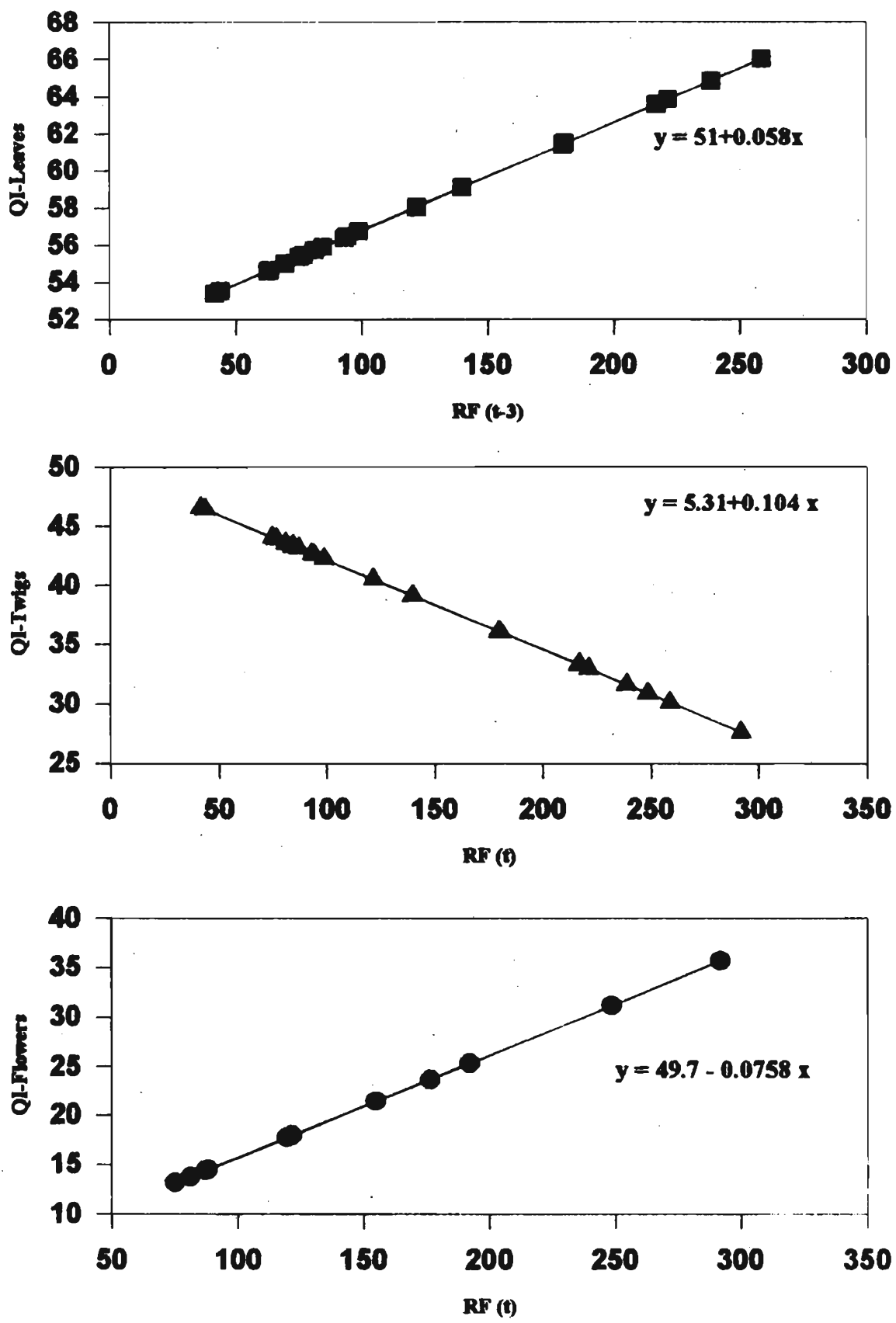

Figure 3 : Linear regression models of rainfall (prelagged 3 months for leaf data) and quiescent infections (QI) of leaves, twigs and flowers expressed as percentages. 


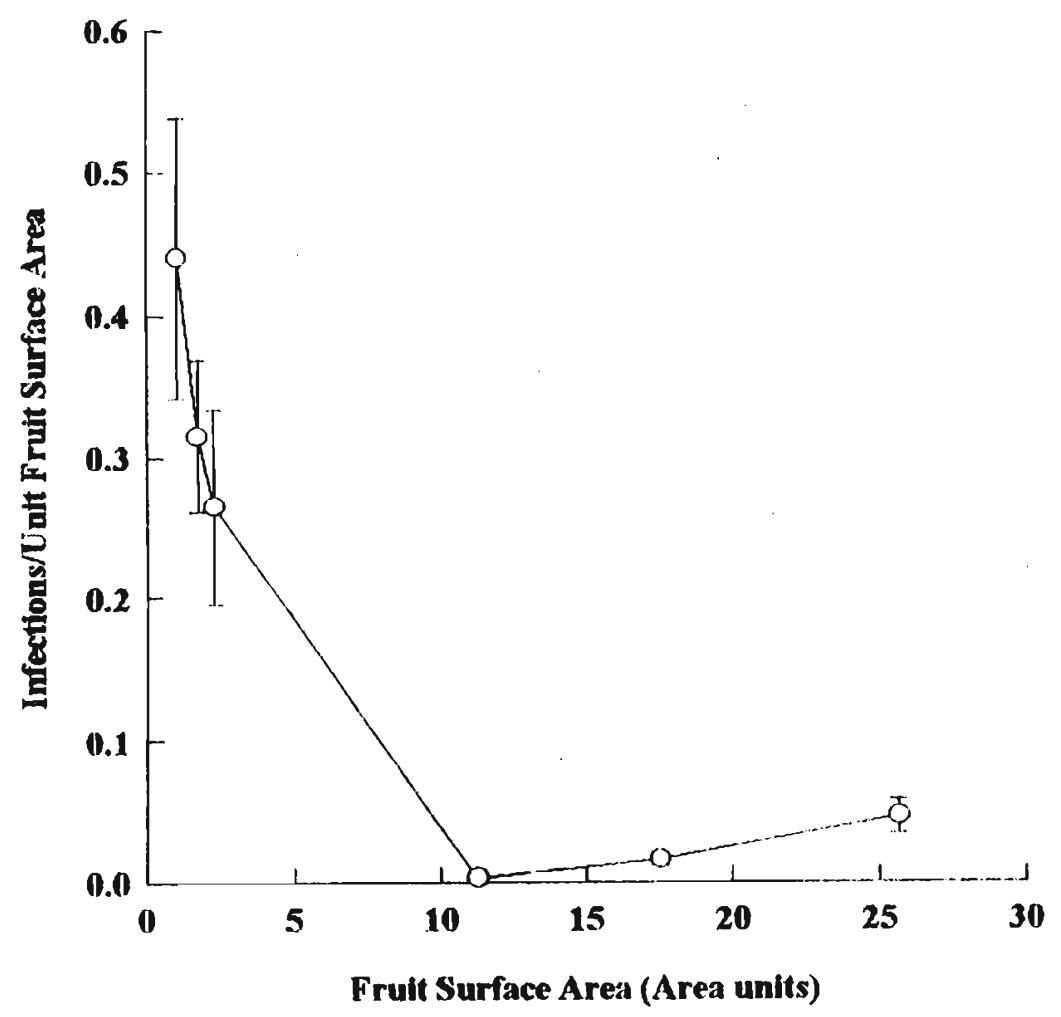

Figure 4: Density of quiescent infections of $C$. gloeosporioides at different development stages of avocado fruit.

\section{DISCUSSION}

The samples for enumerating QI and FLVFP of $C$. gloeosporioides, of leaves, flowers and twigs, were obtained only from the lower branches to eliminate variation caused by sampling from different heights. It is likely that the sunlight falling at different intensities to branches at different heights, among other environmental factors will affect the pathogen counts on branches at different levels of the tree.

The influence of RF on QI in three distinct parts of the tree (i.e. leaves, twigs and flowers) appear to follow three different patterns, making suggestion of control measures (i.e. chemical sprays) complicated. An earlier study on avocado has reported that occurrence of quiescent infections of $C$. gloeosporioides on fruits reached a maximum during the wettest months, but decreased in drier months, and that the data best fitted a non-linear regression model ${ }^{5}$. Although we did not correlate $\mathrm{RF}$ with fruit infections, the statistical relationships of QI observed on other parts of the tree were always linear. 
The positive correlation of QI on flowers and leaves with RF pre-lagged (t-3), and the negative correlations seen with that of twigs and RF, cannot be explained at this stage. The presence of a thick cuticle on avocado leaves may perhaps be a key to explanation of regression coefficient becoming progressively more significant when the RF is lagged from t-3 to t-6 (Table 2).

Initiation of flowering was observed in January and October respectively in the two years (Figure 1), which are dry months. Flowers have remained on the tree for 6 months in 1992, and 8 months in 1993. The QIs on flowers have increased with the rains. The QI of leaves also increased corresponding to RF of 3 months before (Figure 2). Fruit development occurs spread over a period of approximately 4 months during and after the flowering season (unpublished observations of A. M. Karunaratne ). Propagules of C. gloeosporioides either as QI (Figure 1) or FLVFP (Table 1) appear to persist on the tree throughout the year with fluctuating numbers. The C. gloeosporioides inoculum appears to be nurtured by different parts of the tree, each part hosting the propagules through time in a different pattern, although the vegetative parts and flowers are not debilitated. If the pathogen caused disease in these parts the whole tree would get killed eventually, thus preventing a safe niche for the pathogen to survive in between fruiting seasons. This is different to the survival of $C$. glocosporioides causing anthracnose of mango, where the conidia were shown to be produced on leaf lesion $\mathrm{s}^{10}$ throughout the year particularly during flowering. In mango, anthracnose is a devastating field disease in addition to being a postharvest disease.

It is possible that the antifungal compounds reported to be present on leaves ${ }^{1: 3}$ prevent them getting diseased by the pathogen. The role of antifungal compounds on different parts of the tree sustaining the propagules of the pathogen needs a detailed investigation. We have tried inoculating avocado leaves and twigs on cut branches with C. glocosporioides in the laboratory, by keeping the cut-end of the branches fresh, by immersing in water. Conidial suspensions were prepared and drops $(20 \mu l)$ were placed on the upper surface of intact and wounded leaves and horizontal tender twigs, by the method described above for fruits. They were enclosed inside plastic bags containing wet cotton wool to maintain moisture. None produced lesions although the same conidial suspensions were able to produce lesions on wounded spots of fruits as described above (A. M. Karunaratne, unpublished data).

The FLVFP counts observed on the tree was low. The highest counts observed on the tree were $10^{2} \mathrm{CFU} / \mathrm{g}$. A slightly higher value of $10^{3} \mathrm{CFU} / \mathrm{g}$ was observed in the debris. Due to this narrow variation and low counts in the FLVFP, they were not considered in interpreting data. 
The low counts of FLVFP of $C$. gloeosporioides seen in this study may also indicate that the pathogen was unable to cause disease and increase propagule numbers while on the vegetative parts of the tree. Unlike in the present study, in an earlier study on citrus, washings of flower petals have yielded a high number of propagules (i.e. $10^{4}$ to $10^{6}$ per gram fresh weight) of C. gloeosporioides ${ }^{14}$, and this pathogen is known to cause wither tip in citrus, in addition to anthracnose on fruits.

In avocado it is possible that the debris under the tree, acts as the ultimate source of inoculum. The survival of the conidia in the debris under the tree may also explain the spread of the disease to fruits of younger newly grown trees by rain splash from neighbouring older trees. An earlier study on strawberry anthracnose ${ }^{15}$, reported that $C$. acutatum survived in buried strawberry tissue for 9 months, and soil population densities gradually declined over a 11 month period.

As occurrence of anthesis appeared to spread for over a period of four months, we expected that relating the QI on fruits with their age, rather than with RF would give more meaningful data. Since the fruits for analysis were obtained from the same tree, fruit size was taken as a convenient index of maturity in this study although this would not be appropriate in a larger sample where genetical and environmental diversity would have an effect on fruit size. Quiescent infections were higher on fruits soon after anthesis. The levels of antifungal compounds are known to increase gradually and reach a maximum at harvest? ${ }^{7}$. However, there is no evidence that antifungal compounds affect the establishment of quiescent infections.

The significant reduction of the density of quiescent infections when the fruit matures (Figure 4), may be explained by any one or a combination of the following hypotheses: a majority of infections are able to establish at an early stage of fruit maturity because of the peel anatomy; the rate of infection cannot keep up with the log phase of expansion of the fruit to maturity.

Several authors have observed that penetration of $C$. gloeosporioides is inhibited by actively growing host cell ${ }^{16-17}$. A histopathological study of C. gloeosporioides infections to avocado, reported that in fruit inoculated early in development when the epicuticular wax layer was very thin, many appressoria were seen later, resting on top of a thick epicuticular wax layer. In addition, these authors have observed thin infection pegs which appeared to be physically broken in the region of the wax layer directly beneath these appressoria. It is not recorded how many of these detached infection pegs actually were able to cause disease on the ripe fruit. It was observed in the present study and in several other studies, (A. M. Karunaratne, unpublished data), that when intact healthy skin of harvested mature unripe fruits were inoculated with viable $C$. gloeosporioides conidia, lesions did not form in these areas when the fruits ripened. It is possible that the thick wax layer on unripe mature avocados acts as a deterrent to establishment of quiescent infections. 
When fruits at a later stage of development were inoculated with $C$. gloeosporioides infection pegs penetrated the wax layer and once through the wax layer the infection peg became thin and unmelanized ${ }^{2}$. While it is apparent that there is a difference in infection patterns when $C$. gloeosporioides inoculations occur at early stages of fruit development and at later stages, whether both types of infections result in lesion formation is not known. In an earlier study $^{1}$, fruits covered early after fruit set showed no infections or lesions at ripening, caused by $C$. gloeosporioides. However fruits inoculated with $C$. gloeosporioides at different stages of development (as much as 3 months prior to harvest), developed anthracnose lesions at ripening. These authors do not report how many lesions that formed on the fruit could be identified with the inoculations made by them earlier.

Several other environmental factors and physiological condition of the host will govern conidial counts and infections. An earlier study has shown that disease development on two weeds by $C$. gloeosporioides f. sp. malvae was affected by inoculum concentration and plant growth stage at the time of inoculation, post inoculation air temperature and, dew-period duration and temperature ${ }^{16}$.

A study which would establish the presence of antifungal compounds on twigs and flowers and the influence of these compounds in different parts of the tree on the pathogen will help in understanding the pathogenicity of C. gloeosporioides on avocado. Eventually, control measures could be recommended, based on these findings.

\section{Acknowledgment}

Prof. T. R. Swinburne, of Wye College, UK for discussions.

\section{References}

1. Binyamini N. \& Schiffman-Nadel M. (1971). Latent infections in avocado fruits due to Colletotrichum gloeosporioides. Phytopathology 62: 592-594.

2. Coates L. M., Muirhead I. F., Irwin J.A.G. \& Gowanlock D.H. (1993). Initial infection processes by Colletotrichum gloeosporioides on avocado fruit. Mycological Research 97 (11): 1363-1370.

3. Adikaram N.K.B., Ewing D. F., Karunaratne A. M. \& Wijeratne E. M. K. (1992). Antifungal compounds from immature avocado. Phytochemisty 31:93-96.

4. Prusky D., Plumbley R.A. \& Kobiler I. (1991). The relationship between antifungal diene levels and fungal inhibition during quiescent infection of unripe avocado fruits by Colletotrichum gloeosporioides. Plant Pathology 40: $45-52$. 
5. Darwas J. M. \& Kotze J. M. (1987). Avocado fruit diseases and their control in South Africa. South African Avocado Grower's Association Yearbook 10: 117-119.

6. Sivanathan S. (1987). A study of the latent infection of immature avocado fruit by Colletotrichum gloeosporioides. M.Phil thesis, University of Peradeniya, Sri Lanka p. 107

7. Sivanathan S. \& Adikaram N.K.B. (1989). Biological activity of four antifungal compounds in immature avocado. Journal of Phytopathology 125: $97-109$.

8. Peterson R.A. (1978). Susceptibility of Fuerte avocado fruits at various stages of growth to infection by anthracnose and stem-end rot fungi. Australian Journal of Experimental Agriculture and Animal Husbandry 18:158-160.

9. Coates L. M., Irwin J.A.G. \& Muirhead I. F. (1993). The use of a benomyl-reisitant mutant to demonstrate latency of Colletotrichum. gloeosporioides in avocado fruit. Australian Journal of Agricultural Research 44: 763-72.

10. Fitzell R.D. \& Peak C.M. (1984). The epidemiology of anthracnose disease of mango: inoculum sources, spore production and dispersal. Annals of Applied Biology 104:53-59.

11. Prusky D., Fuchs Y. \& Yanko U. (1983). Assessment of latent infections as a basis for control of postharvest disease of mango. Plant Disease 67: 816-818.

12. Chakravarty T. (1957). Anthracnose of banana (Gloeosporium musarum. Cke \& Massee) with special reference to latent infection in storage. Transactions of British Mycological Society. 40: 337-345.

13. Carman R.M. \& Duffield A.R. 1995. The isolation of (R)-2-Hydroxy-4oxohenicosan-1-yl acetate from avocado leaves. Tetrahedron Letters, 36 (12): 2119-2120

14. Agostini J. P. \& Timmer I. W. (1994). Population dynamics and survival of strains of Colletotrichum gloeosporioides on citrus in Florida. Phytopathology 84 (4): 420-425.

15. Eastburn D.M. \& Gubler W. D. (1990). Strawberry anthracnose: detection and survival of Colletotrichum acutatum in soil. Plant Disease 74:161-163. 
16. Makowski R.M.D. (1993). Effect of inoculum concentration, temperature, dew period, and plant growth stage on disease of round-leaved mallow and velvet leaf by Colletotrichum gloeosporioides f. sp. malvae. Phytopathology 83:1229-1234.

17. Stanghellini M.E. \& Aragaki M. (1966). Relation of periderm formation and callose deposition to anthracnose resistance in papaya fruit. Phytopathology 56: $444-450$. 